\title{
Fatores associados à gravidade da morbidade materna na caracterização do near miss
}

\author{
Factors associated with the severity of maternal morbidity \\ for the characterization of near miss
}

João Paulo Dias de Souza ${ }^{1}$, José Guilherme Cecatti ${ }^{2}$, Mary Angela Parpinelli ${ }^{3}$

\section{RESUMO}

Objetivos: aplicar um escore de gravidade em casuística de morbidade materna grave (MMG) e comparar os critérios para classificação. Métodos: estudo caso-controle como análise secundária de casuística de MMG de maternidade terciária em período de 12 meses. Nos casos identificados de MMG, aplicou-se escore específico para graduação da gravidade. Vinte casos de morbidade materna near miss (maior gravidade) foram comparados com 104 controles (menor gravidade) de outras morbidades, quanto a fatores de risco, determinantes primários e demanda assistencial. A análise incluiu o cálculo de médias e proporções, utilizando os testes estatísticos $t$ de Student, Wilcoxon, $\chi^{2}$ e estimativas de OR e IC 95\%. Resultados: a maior gravidade (near miss) foi identificada em $16,1 \%$ da casuística e o antecedente de aborto foi o único fator significativamente a ela associado $(\mathrm{OR}=3,41$; IC $95 \%=1,08-10,79)$. Os indicadores de complexidade de assistência foram de fato mais freqüentes no grupo de near miss, que também apresentou número menor de casos com hipertensão ( $30 \%$ contra $62,5 \%)$ e maior com hemorragia (35,5\% contra 10,6\%) como fatores determinantes primários de morbidade grave. Conclusões: a maior gravidade da morbidade materna associou-se ao antecedente de aborto e à hemorragia como causa. $\mathrm{O}$ escore aplicado conseguiu identificar um subgrupo de maior gravidade (near miss) e que demanda atendimento profissional e institucional mais complexo para evitar a ocorrência do óbito.

PALAVRAS-CHAVE: Complicações na gravidez; Mortalidade materna; Morbidade; Cuidados intensivos

\section{ABSTRACT}

Purpose: to apply a severity score to cases of severe maternal morbidity (SMM) and to compare the classification criteria. Methods: a control-case study was performed as a secondary analysis of cases of SMM in a tertiary level maternity unit for a period of 12-month. A specific score for assessing the degree of severity was applied to cases identified as SMM. Twenty cases of near miss maternal morbidity (higher severity) were compared to 104 control cases (lower severity) of other severe morbidities, regarding risk factors, primary determinants and assistance requirements. Analyses were performed with means and proportions, using Student's $t$, Wilcoxon and $\chi^{2}$ statistical tests, and estimations of OR and $95 \%$ CI. Results: the higher severity (near miss) was identified in $16.1 \%$ of cases and the history of abortion was the only factor statistically associated with it $(\mathrm{OR}=3.41,95 \%$ CI 1.08-10.79). In fact, the indices of assistance complexity were more frequent in the near-miss morbidity group, which also presented less hypertension ( $30 \%$ against $62.5 \%$ ) and more hemorrhage (35.5\% against $10.6 \%$ ) as primary determinant factors of severe morbidity. Conclusions: the higher severity of maternal morbidity was associated with a history of abortion and with hemorrhage as a cause. The applied score was able to identify a higher severity subgroup (near miss), which needs more complex professional and institutional care in order to avoid the occurrence of death.

KEYWORDS: Pregnancy complications; Maternal mortality; Morbidity; Near miss; Intensive care

1 Médico Intensivista e aluno do Programa de Doutorado do Departamento de Tocoginecologia da Faculdade de Ciências Médicas da Universidade Estadual de Campinas - (SP).

2 Professor Associado de Obstetrícia do Departamento de Tocoginecologia da Faculdade de Ciências Médicas da Universidade Estadual de Campinas - (SP). 3 Professora Doutora de Obstetrícia do Departamento de Tocoginecologia da Faculdade de Ciências Médicas da Universidade Estadual de Campinas - (SP). Correspondência: José Guilherme Cecatti

CP 6181 - 13081.881 - Campinas - SP - e-mail: cecatti@unicamp.br 


\section{Introdução}

A morte materna é evento trágico que acomete a mulher durante o processo fisiológico da reprodução. Constitui indicador da iniqüidade existente entre os gêneros e está inversamente associada ao grau de desenvolvimento humano ${ }^{1}$. Além disso, as causas diretas de morte materna representam a maioria dos eventos no Brasil, assim como no mundo, e são evitáveis em sua quase totalida$\mathrm{de}^{2,3}$. Entretanto, este acontecimento ainda é mundialmente negligenciado, sendo que um dos fatores que determinam esta situação é o desconhecimento do tamanho do problema a ser enfrentado ${ }^{4}$. No Brasil a situação é semelhante, e o registro vital incompleto e a subnotificação das causas maternas de morte são elementos que dificultam a contabilização de boa parte dos casos de óbito materno ${ }^{5}$. Neste contexto, a estimativa do número de mortes maternas no Brasil é tema controverso, com números que oscilam entre 56, 160 e 260 mortes maternas para cada 100.000 nascidos vivos ${ }^{6-8}$.

Em decorrência destas dificuldades, e considerando que em países desenvolvidos a morte materna é um evento muito pouco freqüente e que tende a ser cada vez mais raro, o valor da utilização do número de mortes maternas e das informações advindas do seu estudo tem sido reconsiderado. Diante deste quadro, a necessidade de um melhor indicador das condições de saúde materna tem feito crescer o interesse no estudo de casos de mulheres que desenvolveram morbidade grave durante a gestação, parto e puerpério ${ }^{4}$.

$\mathrm{O}$ estudo de mulheres com morbidade materna near miss, ou seja, daquelas que sobrevivem a complicações potencialmente fatais durante a gravidez, parto ou puerpério, é atividade que tem sido vista como promissora para o desenvolvimento deste novo indicador de saúde materna. Este padrão de morbidade entre as mulheres ocorre com mais freqüência do que os casos de óbito materno e é capaz de fornecer informações muito mais completas e consistentes, à medida em que, vivas, são as próprias mulheres que constituem a fonte de informações da problemática enfrentada ${ }^{9}$.

Neste sentido, considerando que a sobrevivência da mulher com morbidade near miss depende, na maioria das vezes, do cuidado oferecido, o estudo mais pormenorizado destes casos também tem sido utilizado para avaliar a qualidade da atenção obstétrica ${ }^{9}$. E, com relação ao próprio cuidado dos casos de maior gravidade, muitos dos estudos têm indicado a transferência da mulher para uma unidade de terapia intensiva clínica ou cirúrgica sem, entretanto, existir consenso sobre o cuidado crítico de mulheres que desenvolvem morbidade materna grave ${ }^{10}$. Analogamente ao que foi descrito para a investigação dos óbitos maternos, a inexistência de planejamento detalhado para a oferta de cuidados pode favorecer a ocorrência de demoras na implementação de medidas necessárias, o que tem sido associado a um desfecho materno-fetal desfavorável ${ }^{11}$.

Entretanto, o conceito de morbidade materna near miss é ainda recente e tem sido utilizado em sentido amplo por diferentes pesquisadores, havendo controvérsia sobre quais critérios indicadores de near miss seriam os mais adequados. Esta controvérsia pode ser atribuída, em parte, à dificuldade de proceder à categorização do espectro de gravidade clínica que possui em suas extremidades, de um lado, a gravidez saudável e, do outro, o óbito materno. Neste continuum insere-se determinado grau de morbidade grave compativel com o conceito near miss ${ }^{12}$.

Recentemente, foi desenvolvido um escore que, a partir de características clínicas e outras relativas ao manejo de mulheres com morbidades maternas graves, busca identificar os casos de near miss, diferenciando-as daquelas mulheres também portadoras de morbidade grave, porém sem ameaça à vida ${ }^{13}$.

O presente estudo realizou a aplicação do referido escore na casuística de morbidade materna grave de um centro terciário de saúde da mulher, comparando os grupos de morbidade materna near miss e de outras morbidades maternas graves quanto a fatores de risco, determinantes primários e sua demanda assistencial.

\section{Métodos}

Foi desenvolvido estudo caso-controle, a partir dos dados obtidos em um estudo de corte transversal realizado na maternidade do Centro de Atenção Integral à Saúde da Mulher (CAISM) da Universidade Estadual de Campinas, no periodo de $1^{\circ}$ julho de 2003 a 30 de junho de 2004. Houve aprovação do Comitê de Ética em Pesquisa da Faculdade de Ciências Médicas da UNICAMP.

Para a constituição do banco de dados foram obtidas informações dos prontuários de mulheres atendidas no CAISM e que desenvolveram morbidade materna grave. Foram consideradas mulheres portadoras de morbidade materna grave aquelas que, durante a gestação, parto ou nos primeiros 42 dias de puerpério, tivessem apresentado quaisquer das condições listadas na Tabela 1 , de acordo com os critérios já amplamente utilizados de Mantel et al. ${ }^{14}$ e Waterstone et al. ${ }^{15}$. De maneira geral, os critérios de Mantel incluem condições características de disfunção orgânica dos mais variados órgãos e sistemas e procedimen- 
tos relacionados ao seu cuidado, ao passo que os critérios de Waterstone incluem o diagnóstico clínico das condições patológicas mais freqüentemente associadas ao óbito materno. Ambos foram utilizados sistematicamente para a busca ativa de casos de morbidade materna grave.

Tabela 1 - Critérios indicativos de morbidade materna grave, segundo Mantel et al. ${ }^{14} \mathrm{e}$ Waterstone et al. ${ }^{15}$.

\begin{tabular}{l} 
Mantel \\
\hline Edema pulmonar \\
Parada cárdio-respiratória \\
Hipovolemia necessitando de cinco ou mais unidades de concentrado \\
de hemácias \\
Admissão em UTI por sepse \\
Histerectomia de emergência por sepse \\
Intubação e ventilação por mais de 60 minutos (exceto durante anestesia \\
geral) \\
Saturação periférica de $\mathrm{O}_{2}<90 \%$ por mais de 60 minutos \\
Relação entre a pressão parcial de oxigênio e a fração inspirada de \\
oxigênio (PaO $/$ FiO ${ }_{2}$ ) <300 mmHg \\
Oligúria (diurese <400 mL/24 h, refratária à hidratação cuidadosa ou à \\
furosemida ou dopamina) \\
Aumento agudo da uréia para 15 mmol/L ou de creatinina >400 mmol/L \\
Icterícia na presença de pré-eclâmpsia \\
Cetoacidose diabética \\
Crise tirotóxica \\
Trombocitopenia aguda requerendo transfusão de plaquetas \\
Coma por mais de $12 \mathrm{~h}$ \\
Hemorragia subaracnóide ou intraparenquimatosa \\
Admissão em UTI por qualquer razão \\
Histerectomia de emergência por qualquer razão \\
Acidente anestésico: ( 1 ) hipotensão grave associada a anestesia \\
epidural ou raquidiana (pressão sistólica < 90 mmHg por mais de 60 \\
min); (2) insucesso na intubação traqueal requerendo a reversão \\
anestésica \\
\hline
\end{tabular}

\section{Waterstone}

Pré-eclâmpsia grave

Eclâmpsia

Síndrome HELLP

Hemorragia grave

Sepse grave

Rotura uterina

A identificação e classificação dos casos de morbidade materna grave aconteceram por meio de visita diária de um dos pesquisadores ao Centro Obstétrico, Unidade de Terapia Intensiva e Enfermaria de Patologia Obstétrica da instituição. Sempre que uma mulher era identificada como tal, o critério reconhecido inicialmente foi o considerado como o de inclusão no estudo. Para todas as mulheres incluídas pela presença de critérios definidos por Mantel, foi também pesquisada, na- quele momento, a coexistência de algum critério de Waterstone, e vice-versa. A equipe responsável pela assistência da mulher não foi comunicada da identificação da mulher como um caso de morbidade materna grave no estudo, a fim de evitar possiveis vieses na conduta.

No presente estudo foram analisados os seguintes dados: idade, paridade, idade gestacional no momento da identificação do caso, tempo de puerpério, antecedente de cesárea, antecedente de aborto, idade gestacional na resolução da gestação, via de parto, tempo de permanência na UTI, tempo de permanência hospitalar total, utilização de hemoderivados e de procedimentos especiais. Os procedimentos especiais foram definidos como intervenções terapêuticas ou propedêuticas que não são habitualmente utilizadas durante a gestação, o parto ou o puerpério, como, por exemplo, acesso venoso central e/ou arterial, cateterismo cardíaco direito, ecocardiografia, ultra-sonografia não obstétrica, histerectomia e ventilação mecânica, entre outros.

Em cada um dos casos foi aplicado o escore de morbidade materna grave de cinco fatores desenvolvido por Geller et al., em $2004^{13}$. Este escore é baseado na presença ou ausência de cinco condições (falência orgânica, admissão em UTI, intubação prolongada por mais de 12 horas, hemotransfusão superior a três unidades e intervenção cirúrgica como conseqüência da morbidade materna apresentada) e foi desenvolvido para classificar os casos de morbidade materna grave em "morbidade materna near miss" (maior gravidade) ou "outra morbidade materna grave" (menor gravidade). De acordo com o exposto na Tabela 2, cada condição clínica acima citada corresponde a uma determinada pontuação e o escore total de cada caso é obtido somando-se a pontuação das condições presentes. As mulheres com escore igual ou superior a oito são consideradas portadoras de "morbidade materna near miss", ao passo que aquelas com pontuação inferior a oito são classificadas como portadoras de "outra morbidade materna grave"13.

Tabela 2 - 0 escore de morbidade materna grave e a pontuação relativa de cada fator.

\begin{tabular}{lc}
\hline Fator & No $^{0}$ de pontos \\
\hline Insuficiência orgânica & 5 \\
Admissão em UTI & 4 \\
Transfusão (>3 unidades) & 3 \\
Intubação prolongada (>12 horas) & 2 \\
Intervenção cirúrgica (conseqüente à morbidade grave) & 1 \\
\hline
\end{tabular}

Obtém-se o escore de cada paciente procedendo-se ao somatório do número de pontos correspondente às condições clínicas apresentadas. Um escore maior ou igual a oito (8) pontos indica a condição "hear-miss". Escores iguais ou inferiores a sete (7) indicam a condição de "outras morbidades graves". Modificado de Geller et al. ${ }^{13}$. Falência cardíaca, pulmonar, hematológica, renal, hepática/gastrointestinal ou do sistema nervoso central. 
Após a aplicação deste escore, a casuística foi dividida em dois subgrupos: mulheres portadoras de morbidade materna near miss (casos) e mulheres portadoras de outras morbidades maternas graves (controles). A seguir, os dados coletados foram comparados e para isso, foi testada a hipótese de normalidade da distribuição dos dados de cada variável nos subgrupos, pelo teste de Kolmogorov-Smirnov. Os dados de distribuição normal foram comparados com o uso do teste $t$ de Student, ao passo que os dados de distribuição livre foram comparados por meio do teste de Wilcoxon-Mann-Whitney. Quando necessário, proporções foram comparadas pelo teste do $\chi^{2}$ clássico. Para os fatores potencialmente associados à gravidade da condição materna, calculou-se o risco estimado mediante o OR e seu respectivo IC 95\%. O suporte computacional foi provido pelos softwares MedCalc (Mariakerke, Bélgica) e EpiInfo.

\section{Resultados}

No período de um ano correspondente à coleta de dados sobre a ocorrência de casos de morbidade materna grave na instituição, 2.929 mulheres tiveram parto, ocorreram duas mortes maternas e 124 mulheres foram classificadas como portadoras de morbidade materna grave pelos critérios de Mantel e de Waterstone. De acordo com o sistema de cinco fatores para avaliação de morbidade materna grave, foram identificados 20 (16\%) como morbidade materna near miss (casos) e 104 (84\%) como outras morbidades graves (controles), com uma proporção de cinco controles para cada caso. Desta forma, foi observada uma razão de morbidade materna grave de 42,3 casos por 1.000 partos, ao passo que a razão de near miss foi de 6,8 casos por 1.000 partos.

As características dos casos clínicos estudados, de acordo com a classificação entre morbidade materna near miss e outra morbidade materna grave, são apresentadas nas Tabelas 3 e 4. Não foi observada diferença estatisticamente significativa entre os subgrupos, no que se refere à idade, via de parto, idade gestacional e antecedente de cesárea.
Tabela 3 - Comparação entre características de mulheres com morbidade near miss (escore de morbidade grave igual ou maior que 8) e mulheres com outras morbidades graves.

\begin{tabular}{|c|c|c|c|}
\hline & \multicolumn{3}{|c|}{ Morbidade Outra morbidade } \\
\hline & near miss & grave & $p$ \\
\hline & Média \pm DP & Média \pm DP & \\
\hline Idade & $29,0 \pm 8,6$ & $27,2 \pm 7,2$ & $0,395^{*}$ \\
\hline Paridade & $2,5 \pm 2,0$ & $1,4 \pm 1,7$ & $0,036^{* *}$ \\
\hline Idade gestacional na identificação & $30,8 \pm 7,1$ & $27,0 \pm 5,4$ & $0,027^{*}$ \\
\hline Tempo de puerpério na identificação & $3,3 \pm 2,2$ & $5,7 \pm 9,6$ & $0,337^{\star *}$ \\
\hline Idade gestacional na resolução & $29,8 \pm 6,7$ & $33,2 \pm 5,7$ & 0,061 \\
\hline Permanência na UTI & $10,2 \pm 13,9$ & $3,5 \pm 1,8$ & $0,044^{* *}$ \\
\hline $\begin{array}{l}\text { Transfusão de concentrado de } \\
\text { hemácias (número de unidades) }\end{array}$ & $7,4 \pm 4,6$ & $3,3 \pm 1,9$ & $0,013^{*}$ \\
\hline Procedimentos especiais (número) & $3,7 \pm 2,3$ & $0,4 \pm 0,8$ & $<0,001^{* *}$ \\
\hline Permanência hospitalar (dias) & $24,2 \pm 28,1$ & $7,6 \pm 4,3$ & $0,016^{* *}$ \\
\hline
\end{tabular}

Tabela 4 - Comparação de proporções das características de mulheres com morbidade materna near miss e com outras morbidades maternas graves.

\begin{tabular}{|c|c|c|c|c|c|c|}
\hline & \multicolumn{2}{|c|}{ Near-miss } & \multicolumn{2}{|c|}{$\begin{array}{c}\text { Outras morbidades } \\
\text { graves }\end{array}$} & \multirow[b]{2}{*}{ OR } & \multirow[b]{2}{*}{ IC $95 \%$} \\
\hline & $\mathrm{n}$ & $\%$ & $n$ & $\%$ & & \\
\hline \multicolumn{7}{|l|}{ Idade } \\
\hline$<20$ & 4 & 20,0 & 15 & 14,4 & 2,10 & $(0,41-8,77)$ \\
\hline $20-34$ & 9 & 45,0 & 71 & 68,3 & 1,00 & - \\
\hline$>34$ & 7 & 35,0 & 18 & 17,3 & 3,07 & $(0,88-10,69)$ \\
\hline \multicolumn{7}{|l|}{ Paridade } \\
\hline 0 & 4 & 20,0 & 38 & 36,5 & 1,00 & - \\
\hline $1-4$ & 14 & 70,0 & 59 & 56,7 & 2,25 & $(0,64-10,05)$ \\
\hline$>4$ & 2 & 10,0 & 7 & 6,7 & 1,20 & $(0,11-7,31)$ \\
\hline \multicolumn{7}{|c|}{ Via de parto* } \\
\hline Vaginal & 6 & 33,3 & 15 & 14,8 & 2,87 & $(0,75-9,82)$ \\
\hline Cesárea & 12 & 66,7 & 86 & 85,1 & 1,00 & - \\
\hline \multicolumn{7}{|c|}{ Idade gestacional* } \\
\hline$<28$ & 3 & 16,6 & 12 & 11,8 & 1,85 & $(0,25-11,13)$ \\
\hline $28-36$ & 10 & 55,5 & 52 & 51,5 & 1,42 & $(0,40-5,74)$ \\
\hline$>36$ & 5 & 27,7 & 37 & 36,6 & 1,00 & - \\
\hline \multicolumn{7}{|c|}{$\begin{array}{l}\text { Antecedentes de } \\
\text { cesárea }\end{array}$} \\
\hline Presente & 9 & 45,0 & 28 & 26,9 & 2,22 & $(0,75-6,58)$ \\
\hline Ausente & 11 & 55,0 & 76 & 73,1 & 1,00 & $(0,40-5,74)$ \\
\hline \multicolumn{7}{|c|}{$\begin{array}{l}\text { Antecedentes de } \\
\text { aborto }\end{array}$} \\
\hline Presente & 8 & 40,0 & 17 & 16,3 & 3,41 & $(1,08-10,79)$ \\
\hline Ausente & 12 & 60,0 & 87 & 83,7 & 1,00 & - \\
\hline Total & 20 & 16,1 & 104 & 83,9 & & \\
\hline
\end{tabular}


As mulheres com morbidade near miss apresentaram paridade maior $(2,5 \pm 2,0)$ que aquelas portadoras de outras morbidades graves $(1,4 \pm 1,7)$. A identificação de mulheres portadoras de morbidade materna near miss ocorreu em idade gestacional mais avançada $(30,8 \pm 7,1$ versus $27,0 \pm 5,4)$. Com relação aos procedimentos especiais, foram realizados, ao todo, 126 procedimentos, sendo 44 entre as mulheres que desenvolveram outras morbidades maternas graves e 82 entre as mulheres com morbidade materna near miss (respectivamente $0,4 \pm 0,8$ versus $3,7 \pm 2,3$ ). A permanência em UTI e a utilização de procedimentos especiais e de concentrado de hemácias foram maiores no grupo de mulheres que foi classificado como portadoras de morbidade materna near miss. Foi observado que o antecedente de aborto esteve estatisticamente associado ao desenvolvimento da morbidade near miss $(\mathrm{OR}=3,41$; IC 95\%=1,08-10,79).

Com relação aos fatores determinantes primários de morbidade grave, observou-se que houve menos casos com hipertensão (30 versus $62,5 \%$ ) e mais casos com hemorragia $(35,5$ versus $10,6 \%)$ no grupo de mulheres que desenvolveu morbidade near miss, ao passo que a freqüência de sepse e das complicações clínicas foi semelhante entre os dois grupos (Tabela 5)

Tabela 5 - Comparação de proporções (\%) de fatores determinantes primários de morbidade grave entre mulheres com near miss e outras morbidades graves.

\begin{tabular}{lccc}
\hline $\begin{array}{l}\text { Fatores determinantes } \\
\text { primários }\end{array}$ & Near miss & $\begin{array}{c}\text { Outras } \\
\text { morbidades }\end{array}$ & $\mathbf{p}^{*}$ \\
\hline Hipertensão & 30,0 & 62,5 & 0,0145 \\
Hemorragia & 35,5 & 10,6 & 0,0125 \\
Sepse & 10,0 & 5,8 & 0,8529 \\
Complicações clínicas & 25,0 & 21,1 & 0,1454 \\
Total & 20 & 104 & \\
\hline
\end{tabular}

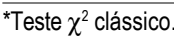

\section{Discussão}

No presente estudo foi observada razão de near miss de 6,8 casos por 1.000 partos (20:2929), que está dentro da ampla margem de razões de near miss descritas na literatura $(0,7-101,7: 1000$ partos), com variações dependentes do local, disponibilidade de recursos, tipo de critérios utilizados para a definição dos casos, entre outros ${ }^{16}$.

Analisando-se o perfil das intervenções terapêuticas requeridas para a assistência de mulheres com morbidade materna grave, observa-se que a maior parte destas mulheres não demandou assistência que envolvesse recursos materiais maiores do que o previsto para a assistência obstétrica de boa qualidade, tendo sido necessária maior vigilância e atenção, o que efetivamente depende da formação do obstetra e das habilidades da equipe. Na presente casuística, cerca de dois terços dos procedimentos especiais foram realizados nas 20 mulheres caracterizadas como apresentando morbidade near miss, sendo este o grupo de maior complexidade e custo.

Por outro lado, demandas propedêuticas e terapêuticas especiais podem representar uma barreira para a assistência de mulheres com complicações graves, especialmente em maternidade ou centros de referência dissociados de hospitais gerais ou em locais com recursos limitados. O conhecimento destas características poderá indicar a gerentes e planejadores medidas visando otimizar a assistência e diminuir a ocorrência de demoras evitáveis.

Em decorrência da dispersão territorial, mesmo o considerável número de mortes maternas que ocorre anualmente no Brasil resulta em eventos pouco freqüentes na maioria das maternidades e hospitais brasileiros. Esta relativa raridade dificulta o estudo da morte materna, bem como, do ponto de vista institucional, complica a análise dos resultados de políticas implementadas para o combate à morte materna.

No presente estudo, houve a ocorrência de apenas dois casos de morte materna, ao passo que puderam ser identificados, por diferentes critérios, 124 casos de morbidade materna grave, sendo que 20 deles foram considerados de morbidade near miss. Aceitando-se a proposição de que, pelo menos em parte, os determinantes da morte materna são comuns aos determinantes da morbidade grave, em especial dos casos de near miss, o estabelecimento de sistema de vigilância epidemiológica baseado na identificação prospectiva dos casos de morbidade grave em ambiente hospitalar possibilitaria o estudo de determinantes da morbimortalidade de uma forma dezenas de vezes mais freqüente, de maneira mais detalhada e com a possibilidade de, em alguns casos, recomendar determinadas intervenções possivelmente capazes de evitar a evolução fatal ${ }^{17}$.

Por outro lado, considerando a definição em si de near miss, verifica-se que uma parcela da controvérsia previamente referida sobre esta definição é atribuível à dificuldade no estabelecimento de qual gravidade, dentro do continuum de morbidade existente entre a gestação normal e o óbito, deve corresponder a esta condição. A maioria dos estudos realizados em países desenvolvi- 
dos tem adotado definições operacionais de near miss baseadas na admissão em UTI ${ }^{16}$. Embora exista esta tendência entre os pesquisadores dos países desenvolvidos, a adoção desta definição poderá não ser adequada para os países em desenvolvimento, como o Brasil, uma vez que a organização de cada serviço, bem como a disponibilidade de leitos de terapia intensiva, podem representar considerável viés, visto que os serviços que dispõem de leitos mais facilmente tendem a realizar a indicação de terapia intensiva de forma mais precoce e às vezes até preventiva, ao passo que serviços com maior limitação de leitos tendem a admitir as mulheres em estado mais grave, muitas vezes quando o processo letal já se encontra deflagrado, resultando em maior mortalidade.

Por outro lado, as definições de near miss baseadas em condições clínicas estão, em geral, centradas nas maiores causas de morte materna: hipertensão, hemorragia e sepse. Contudo, foram observadas diferenças nas definições de cada situação clínica, como, por exemplo, a quantidade de sangue perdido para que a hemorragia seja considerada grave e indicativa de near miss. Outra consideração que deve ser feita é que a capacidade de manejar determinadas complicações não é considerada nestas definições e esta capacidade certamente exerce algum impacto na mortalidade. Assim, à medida que trata de eventos clínicos isolados, que não consideram a resposta orgânica da mulher e do meio, ocorre a tendência de incluir casos mais leves dentre os casos near miss, com isso, talvez, reduzindo o poder desta categoria em compartilhar determinantes comuns da mortalidade materna, o que possivelmente representa um dos maiores objetivos do estudo desta ordem de eventos.

Condições sociodemográficas como o estado marital e a escolaridade já foram associados ao desenvolvimento de morbidade grave ${ }^{17}$. Por outro lado, considerando a presente casuística e utilizando-se o tempo de permanência hospitalar e o grau de intervenções terapêuticas como indicativos da complexidade de manejo e da gravidade clinica, observou-se que as mulheres que foram classificadas como "outras morbidades graves" compuseram um grupo de menor complexidade e gravidade, em comparação com as mulheres que foram classificadas como near miss, informação que pode ser utilizada para subsidiar a aplicação do escore utilizado para identificação dos casos realmente graves, com maior correspondência ao conceito de near miss. Em relação ao achado de maior proporção de hipertensão entre os casos de outras morbidades graves e maior proporção de hemorragia entre os casos near miss, pode ser possivelmente atribuído aos critérios utilizados para compor a amostra, que talvez tenham privilegiado a inclusão de casos menos graves de hipertensão. O antecedente de aborto esteve associado à ocorrência de maior gravidade, entretanto serão necessários mais estudos para que este achado possa ser corroborado e outras inferências sejam feitas.

Finalmente, a despeito das diferentes abordagens e resultados dos estudos, acredita-se que o estudo da morbidade materna grave deverá assumir papel de grande destaque em futuro próximo, como indicador de saúde e balizador de políticas públicas para o combate da morte materna. Neste sentido, ainda que sejam necessários estudos adicionais sobre o assunto, é possivel sugerir que o processo de identificação dos casos de morbidade materna near miss deva ocorrer em duas etapas: a primeira, executada a partir de critérios mais amplos e que atuaria como triagem, identificando os casos de morbidade materna grave candidatos ao status "near miss, e a segunda, baseada em critérios mais rígidos, como aqueles que compõem o escore proposto por Geller et al. ${ }^{13}$, identificaria os casos de near miss. Por fim, acreditamos que a identificação prospectiva em todos os serviços dos casos candidatos ao near miss pode ser a maneira mais efetiva de dar atenção especial e específica a estes casos, evitando que a morbidade grave seja fatal. Para isso, provavelmente a melhor estratégia seja a de utilizar os dois conjuntos de critérios, para dar conta de todas as variações regionais, populacionais, institucionais e profissionais, já que cada conjunto consegue isoladamente identificar um excedente de casos que não é identificado pelo outro conjunto.

Embora atualmente exista essa tendência já comentada da utilização do conceito de near miss na abordagem da morbidade e mortalidade maternas, ainda são poucos os estudos nacionais abordando o problema ${ }^{18-20}$. Nenhum deles mostra panorama de fato representativo de todo o território nacional sobre esta condição.

A presente tentativa de se identificarem fatores que possivelmente estivessem associados à maior gravidade, entre os casos de morbidade materna grave, deverá ser complementada no futuro com estudos prospectivos, de maior casuística e estudando uma gama mais ampla de variáveis que pudessem estar associadas a esta condição, com o objetivo de melhor identificar possiveis marcadores da condição, passiveis de serem triados e de indicarem algum tipo de atenção diferenciada e preventiva. Os determinantes primários de morbidade materna grave mostraram ser os mesmos conhecidos para a morte materna. De qual- 
quer maneira, a aplicação do escore de Geller mostrou ser capaz de identificar de fato um subgrupo de mulheres com morbidade materna grave do tipo near miss em uma população de mulheres brasileiras, para quem vale investir na complexidade do atendimento institucional e profissional, com a finalidade específica de evitar a evolução para morte materna, da mesma forma como ocorreu no estudo original.

\section{Referências}

1. Reduction of maternal mortality: a joint WHO/ UNFPA/UNICEF/World Bank Statement. Geneva: WHO; 1999.

2. Cecatti JG, Albuquerque RM, Hardy E, Faúndes A. Mortalidade materna em Recife: causas de óbitos maternos. Rev Bras Ginecol Obstet. 1998;20(1):7-11.

3. Cecatti JG, Faúndes A, Surita FGC. Maternal mortality in Campinas: evolution, under-registration and avoidance. São Paulo Med J. 1999;117(1):5-12.

4. AbouZahr C. Global burden of maternal death and disability. Br Med Bull. 2003;67:1-11.

5. Ministério da Saúde. Urgências e emergências maternas: guia para diagnóstico e conduta em situações de risco de morte materna. $2^{\mathrm{a}}$ ed. Brasília: Ministério da Saúde; 2000.

6. Fundação Nacional de Saúde (FUNS). Departamento de Informática do SUS (DATASUS). Base de dados sobre saúde de 2002. [citado 2004 abr 4]. Disponivel em: http://www.datasus.gov.br/dirbd/estrut.htm

7. Sociedade Civil Bem-Estar Familiar (BEMFAM). Macro Internacional. Pesquisa nacional sobre demografia e saúde - 1996. Rio de Janeiro: BEMFAM; 1997.

8. WHO. Maternal mortality in 2000: estimates developed by WHO, UNICEF, and UNFPA. Geneva: WHO; 2003.

9. Pattinson RC, Hall M. Near misses: a useful adjunct to maternal death enquiries. Br Med Bull. 2003;67:231:43.
10.Zeeman GG, Wendel GD Jr, Cunningham FG. A blueprint for obstetric critical care. Am J Obstet Gynecol. 2003;188(2):532-6.

11. Maine D, Akalin MZ, Ward VM, Kamara A. The design and evaluation of maternal mortality programs. New York: Columbia University/Centre for Population and Family Health, School of Public Health; 1997.

12. Geller SE, Rosenberg D, Cox SM, Kilpatrick S. Defining a conceptual framework for near-miss maternal morbidity. J Am Med Women Assoc. 2002;57(3):135-9.

13. Geller SE, Rosenberg D, Cox S, Brown M, Simonson L, Kilpatrick S. A scoring system identified near-miss maternal morbidity during pregnancy. J Clin Epidemiol. 2004;57(7):716-20.

14. Mantel GD, Buchmann E, Rees H, Pattinson RC. Severe acute maternal morbidity: a pilot study of a definition for a near-miss. Br J Obstet Gynaecol. 1998;105(9):985-90.

15. Waterstone $\mathrm{M}$, Bewley $\mathrm{S}$, Wolfe $\mathrm{C}$. Incidence and predictors of severe obstetric morbidity: case-control study. BMJ. 2001;322(7294):1089-94.

16. Say L, Pattinson RC, Gulmezoglu AM. WHO systematic review of maternal morbidity and mortality: the prevalence of severe acute maternal morbidity (near miss). Reprod Health. 2004;1(1):3.

17. Geller SE, Rosenberg D, Cox SM, Brown ML, Simonson L, Driscoll CA, et al. The continuum of maternal morbidity and mortality: factors associated with severity. Am J Obstet Gynecol. 2004;191(3): 939-44.

18. Viggiano MGC, Viggiano MB. A necessidade de cuidados intensivos e o termo "near miss mortality" aplicado à realidade brasileira. GO Atual. 2000;9(1):29-32.

19. Viggiano MB, Viggiano MGC, Souza E, Camano L. Necessidade de cuidados intensivos em maternidade pública terciária. Rev Bras Ginecol Obstet. 2004;26(4):317-23.

20. Dias de Souza JP, Duarte G, Basile-Filho A. Near-miss maternal mortality in developing countries. Eur J Obstet Gynecol Reprod Biol. 2002;104(1):80. 